\title{
PERCEPCIÓN DE LOS PROFESORES DEL NIVEL PRIMA- RIA RESPECTO A LA CIUDADANÍA DIGITAL
}

\section{PERCEPTION OF PRIMARY LEVEL TEACHERS REGAR- DING DIGITAL CITIZENSHIP}

\author{
Dr. Sergio Humberto Quiñonez Pech ${ }^{1}$, Dra. Gladis Ivette Chan Chi ${ }^{2}$ \\ 1.* Universidad Autónoma de Yucatán, Yucatán, México. \\ Email: sergio.quinonez@correo.uady.mx (iD https://orcid.org/0000-0001-5220-9912 \\ 2. Universidad Autónoma de Yucatán, Yucatán, México. \\ Email: ivette.chan@correo.uady.mx (D) https://orcid.org/0000-0001-7885-8136
}

Como Citar: Quiñonez Pech, S. H., \& Chan Chi, G. I. (2021). Percepción de los profesores del nivel primaria respecto a la ciudadanía digital. Revista Publicando, 8(28), 37-46. https://doi.org/10.51528/rp.vol8.id2153

\section{RESUMEN:}

En la actualidad, el uso constante de las Tecnologías de Información y Comunicación (TIC) y de internet ha requerido que profesores y estudiantes desarrollen nuevas competencias; así mismo ha surgido la necesidad de que se capaciten continuamente en la implementación de una forma responsable de las tecnologías y por ende fomentar una conciencia de ciudadanía digital. Por lo antes mencionado, este artículo tiene como objetivo describir los resultados de una encuesta sobre el nivel de ciudadanía digital que tienen los profesores de primaria a partir de los diversos aspectos que la integran. El estudio tuvo un enfoque cuantitativo, descriptivo y transversal con una técnica para la recolección y análisis de los datos tipo encuesta. Los participantes fueron 154 profesores del nivel primaria, provenientes de diversas instituciones educativas del municipio de Mérida, Yucatán. Como resultados importantes se puede destacar que el $70 \%$ de los aspectos de la ciudadanía digital fueron valorados en un nivel medio y un $20 \%$ en un nivel bajo. Asimismo, se evidenció con base en las variables edad y grado máximo de estudios que no existe diferencia significativa entre las percepciones de los profesores respecto a la ciudadanía digital. Entre las principales conclusiones está que las TIC son herramientas muy importantes para la enseñanza, ya que ayudan a despertar el interés por aprender y brinda muchos recursos de apoyo, es por lo anterior que es necesario formar tanto a los profesores como a los estudiantes en su uso ético y responsable para construir y consolidar su ciudadanía digital.

\section{Palabras Clave:}

TIC, Ciudadanía digital, Enseñanza primaria.

\section{ABSRTACT:}

Currently, the constant use of Information and Communication Technologies (ICT) and Internet have required teachers and students to develop new skills; likewise, the need has arisen for them to be continuously trained in the responsible implementation of technologies and therefore to promote an awareness of digital citizenship. Due to the aforementioned, this article aims to describe the results of a survey on the level of digital citizenship that primary school teachers have from the various aspects that integrate it. The study had a quantitative, descriptive and cross-sectional approach with a technique for collecting and analyzing survey-type data. The participants were 154 primary level teachers, from various educational institutions in the municipality of Mérida, Yucatán. As important results, it can be highlighted that $70 \%$ of the aspects of digital citizenship were valued at a medium level and $20 \%$ at a low level. Likewise, it was proved based on the variable ages and maximum degree of studies that there is no significant difference between the teachers' perceptions regarding digital citizenship. Among the important conclusions is that ICTs are very important tools for teaching, since they help awaken the interest to learn and provide many support resources, which is why it is important to train both teachers and students in their ethical and responsible use for building and consolidating their digital citizenship.

\section{KEYWORDS:}

ICT, Digital citizenship, Primary education. 


\section{INTRODUCCIÓN}

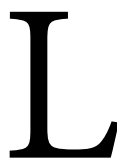

a educación tiene como principal intención la formación integral de los seres humanos, favoreciendo la adquisición y construcción de los conocimientos, el desarrollo de las habilidades, estrategias, así como las actitudes enfocadas en promover el desarrollo de la dimensión ética y los valores (Vincezi y Tudesco, 2009). Es por esto que la educación debe encaminarse hacia la formación humana de una manera articulada, sistemática, intencional y con el apoyo de los diferentes recursos y herramientas que proporcionan las tecnologías de información y comunicación (TIC) (Stramiello, 2005).

En este sentido, Hernández (2017) señala que la tecnología se ha convertido en un catalizador de oportunidades para innovar y apoyar en soluciones de problemas sociales-educativos, por lo cual en el Plan Sectorial de Educación 2020-2024, se establece la importancia que tiene el hecho de disminuir la brecha digital, fomentando la adquisición de los conocimientos, habilidades y responsabilidades necesarias para el uso de las TIC.

Desde esta perspectiva, Ávila (2016) afirma que la educación desempeña un papel crucial en la formación de la ciudadanía digital, además representa un reto indiscutible, ya que el uso de las tecnologías en el campo educativo conlleva al desarrollo de nuevas prácticas de enseñanza-aprendizaje, lo cual permea sustancialmente en el rol del profesorado sin dejar exento a los estudiantes.

La ciudadanía digital inmersa en el campo educativo conlleva la integración de las TIC al servicio de la instrucción, así como hacia la revisión de las dinámicas referentes a las formas de enseñar y aprender utilizando las tecnologías, por lo tanto promover la construcción de una ciudadanía digital implica comprender las formas de participación, respeto, intercambio, colaboración y convivencia en los entornos digitales para construir una sociedad a través de internet (Cobo, 2019) donde pueden desarrollarse interacciones armoniosas entre todos los integrantes de la misma para continuar aprendiendo y formándose como ciudadanos críticos, reflexivos, democráticos e íntegros, capaces de discernir la información que consumen de la internet para compartirla de forma ética y transparente.

En esta misma línea Peña (s/f) argumenta que es debido al masivo acceso a internet, a sus recursos y a los contenidos que ofrece, que tanto los sistemas educativos como los padres de familia tienen que hacerle frente al desafío que conlleva las nuevas formas de aprender y de enseñar a vivir en una sociedad denominada en red, así como en una cultura digital que se encuentra influenciada por los medios digitales, comunidades y grupos sociales que interactúan en línea a través de internet.

De acuerdo con la UNESCO (2014), la ciudadanía digital resulta ser un conjunto de competencias que les permiten a los ciudadanos acceder, recuperar, comprender, evaluar, utilizar, crear y compartir información y contenidos utilizando distintas herramientas y recursos, de manera crítica, ética y eficaz; según Lozano y Fernández (2019), un ciudadano digital es aquella persona que posee la capacidad para ejercer sus derechos de ciudadanía en internet, abocándose a los principios éticos, críticos y coherentes, que promueve el cambio social.

Considerando lo antes mencionado, la escuela debe asumir el reto de formar ciudadanos digitales éticos en el uso de las TIC, así como en el manejo de la información, en su distribución de forma segura, responsable, reflexiva y crítica al mismo tiempo que se centren en promover la alfabetización digital, en disminuir las brechas digitales y favorecer la sana convivencia e interacción desde las redes y entornos virtuales.

Aunado a lo anterior, es importante considerar que uno de los roles principales para la innovación y transformación dentro de las instituciones educativas es el del profesor, ya que él es un agente educativo y socializador; además apoya a los estudiantes en la construcción activa de sus conocimientos, a través de su guía, dirección y orientación. Al mismo tiempo, construye procesos de interacción favorables para la potencialización de las habilidades de los educandos; según Prieto (2008) es quién se encarga de transmitir a través del ejercicio de su docencia una serie de valores enfocados en contribuir de manera directa o indirecta con la formación de los estudiantes.

Ante esto, se puede afirmar que el profesorado ejerce una función esencial dentro del proceso de enseñanza-aprendizaje, puesto que debe asumir el compromiso con los estudiantes de educarlos en la libertad de pensamiento, así como promover en ellos la adquisición de un amplio bagaje de conocimientos que promueva sus actitudes críticas (Prieto, 2008); al mismo tiempo debe de centrar sus esfuerzos en formarlos para convertirlos en ciudadanos éticos y competentes en un ambiente digital.

A partir de lo anterior Galindo (2009), señala que cuando el profesor se encuentra frente a la tecnología adquiere el rol de ciudadano digital, pues debe fortalecer a través de la formación continua el uso ético de las TIC; lo cual representa el punto de partida para llevar a cabo el proceso de humanización de las tecnologías, esto apoya a una implementación de las TIC responsablemente desde los escenarios tecnócrata, reformista y holista, tanto dentro como fuera del aula.

Siguiendo con este mismo orden de ideas, el profesor es 
un agente de cambio, capaz de responder a las demandas de la sociedad digitalizada, puesto que el rápido avance de las TIC ha favorecido el desarrollo de nuevos procesos de enseñanza-aprendizaje; según Antúnez, González, Soler, Rodríguez y Hauß (2013), de nuevas formas para crear, expresar y acceder a diferentes contenidos, de tal forma que, en el mundo globalizado, con mayor frecuencia se hace necesario el uso de los recursos tecnológicos para el tratamiento, análisis y difusión de la información en el ciberespacio; esto ha generado prácticas políticas y sociales a través de internet y los medios digitales para hacer valer los derechos, realizar demandas sociales, exigir transparencia, así como la rendición de cuentas, con el fin de demostrar cómo la sociedad se apropia de lo público para ejercer ciudadanía digital (Alejandro, Benítez y Ortiz, 2015).

La educación no está exenta de lo antes mencionado, la incorporación de las TIC ha causado una revolución en los procesos de enseñanza-aprendizaje, convirtiéndose en un factor para innovar en el sistema educativo, promoviendo prácticas de enseñanza vinculadas al uso de las tecnologías y a la construcción de modalidades no convencionales de aprendizaje. Considerando esto, se hace necesario redireccionar el proceso formativo de los profesores y estudiantes incorporando aspectos vinculados a la era de la digitalización, específicamente educándolos para construir y consolidar su ciudadanía digital.

De aquí la importancia de abordar la ciudadanía digital en el campo educativo, a fin de favorecer desde la escuela la formación de ciudadanos digitales que puedan adquirir conocimientos y desarrollar habilidades que les permitan innovar en el proceso de enseñanza-aprendizaje en los entornos virtuales, incorporando el uso de los diferentes recursos que proporcionan las TIC y la internet de una forma segura, confiable y ética para garantizar su formación integral, ya que de acuerdo con García-Valcárcel, Basilotta y Mulas (2016), la ciudadanía digital, es un espacio en el que las personas pueden relacionarse e interactuar entre sí y con los demás seres humanos que conforman la sociedad, a través del uso potencial que ofrecen las tecnologías de la información y comunicación.

En México, desde el año 2009 se tienen registros del surgimiento de las primeras experiencias visibles de ciudadanía digital, concibiendo al ciudadano digital como aquella persona experta y que es capaz de utilizar de forma responsable las TIC para dar cumplimiento a los objetivos planteados (Walter, 2015; Alva, 2019).

Ante esto, Cárdenas (2018), señala que la Secretaría de Educación Pública en México, estableció como una de sus prioridades impulsar estrategias orientadas a la mejora de la calidad educativa, declarando como uno de los ejes centra- les la incorporación de prácticas y apoyos enfocados hacia una educación innovadora, haciéndose necesario identificar los desafíos referentes a la inclusión de tecnologías en beneficio de la educación en México, de tal forma que se realizó un estudio centrado en diagnosticar puntualmente el nivel de conocimiento tanto de estudiantes como de docentes de las escuelas públicas en ciudadanía digital. Entre los resultados encontrados estaba que los niveles de competencia de los docentes, estudiantes y padres de familia eran similares en cuanto a la ciudadanía digital. Asimismo, se encontró que existía la necesidad de una mayor capacitación de los profesores y padres de familia respecto al uso de las TIC en comparación con los estudiantes, lo cual mostró que los programas relacionados con la alfabetización digital debían expandirse y ser incluyentes.

Por otro lado, en el estado de Yucatán, el Instituto Nacional Electoral (2020) desarrolló el proyecto denominado ciudadanía digital, el cual busca promover la participación ciudadana mediante el uso de las redes sociales; al mismo tiempo pretende fomentar el pensamiento crítico y uso responsable de la información que se comparte y consume a través de internet, esto con el fin de crear conciencia acerca de las interacciones que se realizan con las demás personas de forma individual y colectiva.

En síntesis, se puede afirmar que cuando una persona navega en el ciberespacio esta debe de ser responsable. Por tal motivo para garantizar el éxito de la ciudadanía digital es significativo el uso ético de la información y las TIC; así como promover acciones que favorezcan el acceso de la población a las tecnologías, a fin de combatir la brecha digital y que la escuela como institución formadora logre construir una verdadera cultura digital en la nueva era del conocimiento. Considerando lo antes mencionado, surge la necesidad de identificar el nivel de percepción que tienen los profesores de primaria respecto a su ciudadanía digital con el fin de proponer recomendaciones que permitan el desarrollo de dicha competencia.

\section{MÉTODO}

El estudio se basó en un enfoque cuantitativo con un diseño del estudio descriptivo, ya que sólo se describió las situaciones o eventos relacionados con las variables estudiadas (Hernández, Fernández y Baptista, 2013); también se consideró transversal, puesto que la medición y recolección de los datos se realizó en un sólo momento en el tiempo (Creswell, 2012). La técnica para la recolección y análisis de los datos fue de tipo encuesta, ya que esta permitió describir la percepción de los participantes en la investigación, mediante el registro y análisis de los datos que estos proporcionaron (Isaac y Michael, 1995). Las fases que integran 
el estudio se encuentran articuladas de manera secuencial, permitiendo de esta forma confiabilidad y validez en los resultados obtenidos del análisis de los datos (ver Figura 1).

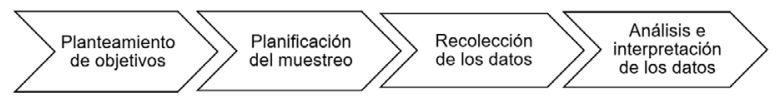

Figura 1. Proceso de la técnica tipo encuesta. Fuente: Arnau, 1995.

Siguiendo las fases de la técnica tipo encuesta, en la primera se planteó el objetivo que guiaría el estudio: describir la percepción que tienen los profesores del nivel primaria respecto a su ciudadanía digital.

Para la segunda fase -selección de los participantes-, se utilizó una muestra no probabilística de tipo intencional (Casal y Mateu, 2003), ya que solamente se tomaron en cuenta a los profesores del nivel primaria del municipio de Mérida, Yucatán, que tuvieron la disponibilidad de contestar el cuestionario que se les administró de manera presencial.

La muestra final estuvo comprendida por 154 profesores del nivel primaria, de los cuales el $73 \%(n=113)$ fueron mujeres y el $26 \%(n=41)$ fueron hombres. Las edades estuvieron comprendidas entre 23 y 57 años $(M=33.79$; $\mathrm{D}$. $\mathrm{T}=$ 9.64).

En la tercera fase -recolección de datos-, cabe mencionar que previamente se realizó una revisión de la literatura, en la cual se destaca el uso estratégico de las TIC y la importancia de la ciudadanía digital. Dicha revisión sirvió para el diseño y elaboración del cuestionario denominado "Diagnóstico de la competencia digital en el nivel básico"; dicho instrumento fue un medio importante que permitió valorar de forma organizada los indicadores de las variables implicadas en el objetivo del estudio (Casas, Repullo y Donado, 2003).

El cuestionario se conformó de dos secciones, la primera tuvo el objetivo de solicitar información general como: sexo, edad, grado de estudios máximos, años de experiencia docente, etc. La segunda sección tuvo como objetivo conocer la percepción del profesor de enseñanza primaria respecto a su competencia digital, a través de 35 reactivos divididos en cuatro dimensiones:

1. Diseño de ambientes de aprendizaje. El profesor cuenta con la capacidad de organizar y gestionar las tecnologías y los espacios digitales haciendo un uso responsable de estos elementos (UNESCO, 2016).

2. Ciudadanía Digital. El profesor crea experiencias para que los estudiantes hagan contribuciones positivas y socialmente responsables, al tiempo que exhiben conductas empáticas en línea para construir relaciones y comunidad (ISTE, 2017).

3. Actualización y colaboración. El profesor dedica tiempo a planificar la colaboración con colegas para crear experiencias auténticas de aprendizaje que aprovechen las TIC (ISTE, 2017).

4. Habilidades técnicas. El profesor aprovecha los principales medios tecnológicos de su entorno (Cota, 2017).

La escala que se utilizó en el instrumento fue tipo Likert, con las siguientes opciones de respuestas del 1 al 4, siendo 1(nunca), 2 (pocas veces), 3 (casi siempre) y 4 (siempre). Este tipo de escalas constituye uno de los formatos más utilizados cuando se desea preguntar varias cuestiones que comparten las mismas opciones de respuesta (Cea D’Ancona, 2001).

Como parte del análisis psicométrico efectuado al cuestionario para determinar su confiabilidad y validez, se realizó la prueba de discriminación de reactivos comparando las puntuaciones en cada uno de estos. Con base en los datos obtenidos, se determinó que a los participantes cuyas puntuaciones estuvieran por arriba del cuartil 75 se les consideraría un adecuado ciudadano digital; en cambio, si obtenían puntuaciones ubicadas por debajo del cuartil 25, se les consideraría limitados en cuanto a su ciudadanía digital. Es importante aclarar que para el objetivo de este estudio solamente se trabajó con la dimensión de ciudadanía digital.

Este proceso estadístico se realizó a través de una prueba t para muestras independientes (ver Tabla 1).

Tabla 1. Prueba de discriminación de reactivos.

$\begin{array}{lll}\text { Reactivos } & t & \mathrm{p} \\ \text { Ciudadanía Digital } & & \end{array}$

Verifico la calidad de la infor-

$-5.324$

0.000 mación encontrada en línea, tomando en cuenta el autor, objetividad, actualidad y veracidad (base de datos, revistas).

Respeto los derechos de autor $-6.052$ 0.000 en la realización de sus materiales docentes (en información, imágenes $\mathrm{y}$ audios).

Promuevo en los estudiantes la búsqueda de información en diversas fuentes confiables en línea. 


\begin{tabular}{lll} 
Reactivos & $\mathrm{t}$ & $\mathrm{p}$ \\
Ciudadanía Digital & & \\
\hline
\end{tabular}

Asesoro a los estudiantes para

$-11.030 \quad 0.000$

que sus búsquedas en línea sean seguras y citen las fuentes consultadas.

Enseño a proteger la información en los ambientes digitales a los estudiantes.

Uso responsablemente las TIC como medio de comunicación interpersonal en grupos (chat, foros...).

Promuevo buenas prácticas y normas de netiquetas en las interacciones sociales de los estudiantes.

Promuevo la cultura colaborativa y libre de discriminación.

Uso software de seguridad y antivirus para prevenir amenazas de internet.

Evito compartir noticias falsas en internet.

Fuente: Elaboración propia.

Del análisis de los datos observados en la Tabla 1, se determinó que los reactivos sí discriminan, ya que el valor de significancia (p) es menor que 0.05. Así mismo, se corrió la prueba estadística denominada alfa de Cronbach y se obtuvo un valor del $a=0.815$, este resultado demostró la confiabilidad del cuestionario. Al respecto, George y Mallery (2003) señalan que un coeficiente de alfa igual o mayor a 0.8 indica que el instrumento es confiable.

Respecto a la fase de recolección de los datos, esta se realizó en las instalaciones asignadas por las instituciones educativas y fue por medio del cuestionario denominado Diagnóstico de la competencia digital en el nivel básico, el cual se administró en un solo momento y fue contestado en formato de papel y lápiz. El proceso contó con la colaboración de los profesores del nivel primaria, los becarios y el responsable de la investigación. El responsable de la investigación explicó lo referente al consentimiento informado, es decir se les comunicó que la información obtenida sería confidencial, anónima y su utilización sería únicamente para los fines establecidos para la investigación; se les mencionó también cuál era el objetivo del cuestionario y se les solicitó a los profesores de primaria su total sinceridad para cada una de las respuestas ofrecidas. Los becarios fueron los encargados de proporcionar los cuestionarios y explicar las instrucciones generales y específicas para contestarlos; de igual manera, el responsable de la investigación siempre estuvo pendiente de las dudas o comentarios que pudieran surgir durante la administración del cuestionario.

En la cuarta fase se realizaron pruebas estadísticas como la obtención de la media, los porcentajes y frecuencias de los datos, esto con el objetivo de categorizar el nivel de la competencia digital; también se realizó la prueba t de student para muestras independientes, para determinar si existía o no diferencias entre la ciudadanía digital y la variable sexo. Por último, se realizó un análisis de varianza para determinar si existía diferencia entre la ciudadanía digital y las variables edad y grado máximo de estudios.

\section{RESULTADOS}

A continuación, se describen las respuestas que dieron las personas encuestadas en el estudio. En una primera instancia y con el objetivo de enriquecer el análisis de los resultados se evidenció que un $89 \%$ de los profesores poseen principalmente una computadora portátil y el equipo tecnológico que menos poseen es el teléfono inteligente con un 54\% (ver Figura 2).

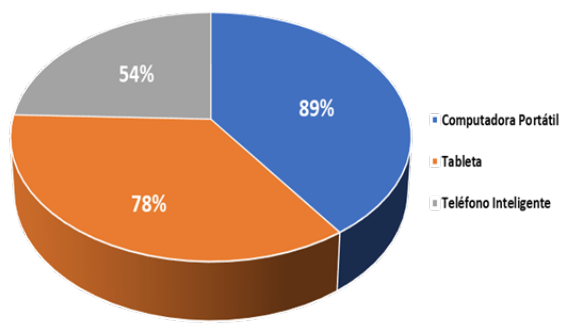

Figura 2. Tipo de equipo que poseen los profesores del nivel primaria. Fuente: elaboración propia.

Así mismo, respecto a los lugares en donde el profesor tiene acceso a internet para realizar sus actividades académicas, se puede observar en la Figura 3, que el 91\% tiene acceso desde su casa y en un menor porcentaje acude al cibercafé para tener servicio a internet.

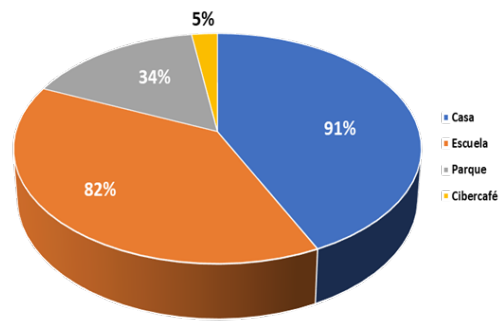

Figura 3. Lugares en los cuales los profesores del nivel primaria tienen acceso a internet. Fuente: elaboración propia. 
Respecto al grado máximo de estudios, se puede observar en la Tabla 2 que existe una diferencia entre el grado de estudios de los profesores del nivel primaria, ya que un $78.5 \%(n=121)$ tienen el grado de licenciatura y sólo el $0.6 \%$ $(n=1)$ tiene estudios de post doctorado. Esto se debe en gran medida, a que los profesores que imparten clases en los niveles básicos provienen de las escuelas normales del país.

Tabla 2. Número de profesores del nivel primaria según el grado máximo de estudios.

\begin{tabular}{llcccccccccc}
\hline Sexo & \multicolumn{1}{c}{ Grado máximo de estudios } & & & Total \\
& Bachillerato & Licenciatura & Maestría & Doctorado & Post Doctorado \\
& f & $\%$ & f & $\%$ & f & $\%$ & f & $\%$ & f & $\%$ & \\
\hline Hombre & 3 & 2 & 32 & 20.7 & 6 & 4 & 0 & 0 & 0 & 0 & 41 \\
Mujer & 3 & 2 & 89 & 57.8 & 18 & 11.6 & 2 & 1.3 & 1 & 0.6 & 113 \\
Total & 6 & 4 & 121 & 78.5 & 24 & 15.6 & 2 & 1.3 & 1 & 0.6 & 154 \\
\hline
\end{tabular}

Fuente: elaboración propia.

Teniendo en retrospectiva las respuestas de los profesores del nivel primaria, se presentan los resultados estadísticos de la dimensión ciudadanía digital (CD); para esto se realizó un análisis de frecuencia y porcentaje de los aspectos que integraban dicha dimensión. El resultado obtenido se analizó teniendo en cuenta el siguiente criterio; si el indicador de valoración obtenido de la suma de los porcentajes de respuestas de la escala alta (casi siempre y siempre) estaban entre $0-60 \%$ se consideraba un nivel de CD bajo, entre 61$79 \%$ un nivel de CD medio y entre un $80-100 \%$ un nivel de CD alto (ver Tabla 3).

Tabla 3. Análisis de frecuencias y porcentajes de la dimensión ciudadanía digital

\begin{tabular}{lcc}
\hline Aspectos & $\begin{array}{l}\Sigma \% \text { Esca- } \\
\text { la alta }\end{array}$ & $\begin{array}{l}\text { Nivel de } \\
\text { CD }\end{array}$ \\
\hline $\begin{array}{l}\text { Asesoro a los estudiantes para } \\
\text { que sus búsquedas en línea sean } \\
\text { se-guras y citen las fuentes consul- } \\
\text { tadas. }\end{array}$ & 60 & Bajo \\
$\quad \begin{array}{l}\text { Uso software de seguridad y } \\
\text { antivirus para prevenir amenazas } \\
\text { de internet. }\end{array}$ & 60 & Bajo \\
$\quad \begin{array}{l}\text { Enseño a proteger la infor- } \\
\text { mación en los ambientes digitales a } \\
\text { los estudiantes. }\end{array}$ & 62 & Medio \\
$\quad \begin{array}{l}\text { Evito compartir noticias falsas } \\
\text { en internet. }\end{array}$ & 62 & Medio
\end{tabular}

\begin{tabular}{lcc}
\hline Aspectos & $\begin{array}{c}\Sigma \% \text { Esca- } \\
\text { la alta }\end{array}$ & $\begin{array}{l}\text { Nivel de } \\
\text { CD }\end{array}$ \\
\hline $\begin{array}{l}\text { Promuevo la cultura colaborati- } \\
\text { va y libre de discriminación. }\end{array}$ & 65 & Medio \\
$\quad \begin{array}{l}\text { Promuevo en los estudiantes la } \\
\text { búsqueda de información en div- } \\
\text { er-sas fuentes confiables en línea. }\end{array}$ & 75 & Medio \\
$\quad \begin{array}{l}\text { Verifico la calidad de la in- } \\
\text { formación encontrada en línea, } \\
\text { tomando en cuenta el autor, } \\
\text { objetividad, actualidad y veracidad } \\
\text { (base de datos, revistas). }\end{array}$ & 76 & Medio \\
$\quad \begin{array}{l}\text { Uso responsablemente las TIC } \\
\text { como medio de comunicación } \\
\text { inter-personal en grupos (chat, } \\
\text { foros...). }\end{array}$ & 78 & Medio \\
$\quad \begin{array}{l}\text { Respeto los derechos de autor en } \\
\text { la realización de sus materiales do- } \\
\text { centes (en información, imágenes } \\
\text { y audios). }\end{array}$ & 79 & Medio \\
$\begin{array}{l}\text { Promuevo buenas prácticas } \\
\text { y normas de netiquetas en las } \\
\text { interac-ciones sociales de los } \\
\text { estudiantes. }\end{array}$ & & \\
\hline
\end{tabular}

Fuente: elaboración propia.

Como se puede observar en la Tabla 3, el 70\% de los aspectos de la dimensión ciudadanía digital fueron valorados en un nivel medio, el $20 \%$ en un nivel bajo y el $10 \%$ en un 
nivel alto.

A partir del resultado obtenido, es importante que los profesores desarrollen los aspectos de la ciudadanía digital referente al asesoramiento de estudiantes para que estos puedan realizar búsquedas en internet confiables y seguras; así mismo, los guíe para que puedan citar dicha información de manera correcta en sus diversos trabajos académicos. Otro aspecto importante por mejorar es el relacionado con el uso del software de seguridad para proteger la información y evitar amenazas que puedan surgir de internet. También es necesario fomentar el avance de los aspectos que fueron valorados en un nivel medio, con el objetivo de una mejora continua y consolidar la formación de la ciudadanía tanto en los profesores como en los estudiantes.

En una tercera fase del análisis cuantitativo, se presenta a continuación la comparación de las dimensiones ciudadanía digital con base en la variable sexo.

Tabla 4. Comparación de la dimensión ciudadanía digital con la variable sexo.

\begin{tabular}{llll}
\hline Dimensión & $\mathrm{t}$ & $\mathrm{gl}$ & $\mathrm{p}$ \\
\hline Ciudadanía Digital & 2.175 & 151 & .031 \\
\hline
\end{tabular}

Fuente: elaboración propia.

Con base en los datos de la Tabla 4, podemos observar que se encontró diferencia entre las medias de los dos grupos, tanto los hombres como las mujeres puntúan de manera diferente en la dimensión de ciudadanía digital; ya que el valor de $\mathrm{p}<.05$.

Teniendo en cuenta los resultados anteriores, se pudo confirmar que los profesores (hombres y mujeres) del nivel básico, tienen diferencias en su percepción respecto a su ciudadanía digital. Siguiendo con la misma línea de análisis cuantitativos, se realizó un análisis de varianza en la cual se comparó la variable edad con la dimensión ciudadanía digital.

Tabla 5. Análisis de la variable edad con la dimensión ciudadanía digital.

\begin{tabular}{lll}
\hline Dimensión & $\mathrm{F}$ & $\mathrm{p}$ \\
\hline Ciudadanía Digital & .540 & .707 \\
\hline
\end{tabular}

Fuente: elaboración propia.
Del análisis de la Tabla 5 se obtuvo que la variable edad no mostró influencia sobre la dimensión ciudadanía digital, ya que el valor de p >.05; es decir los profesores jóvenes y los de mayor edad, evidenciaron no tener diferencia en su percepción respecto a los diversos aspectos de la ciudadanía digital.

También se realizó el análisis de varianza en la cual se comparó la variable grado académico con la dimensión ciudadanía digital.

Tabla 6. Análisis de la variable grado máximo de estudios con la dimensión ciudadanía digital.

\begin{tabular}{lll}
\hline Dimensión & $\mathrm{F}$ & $\mathrm{p}$ \\
\hline Ciudadanía Digital & 1.966 & .103 \\
\hline
\end{tabular}

Fuente: elaboración propia.

Como se puede observar en la Tabla 6, se obtuvo que la variable grado máximo de estudios tampoco mostró influencia sobre la dimensión ciudadanía digital, ya que el valor de $\mathrm{p}>.05$; es decir los profesores con un nivel de estudios de bachillerato hasta postdoctorado, evidenciaron no tener diferencia en su percepción respecto a los diversos aspectos de la ciudadanía digital.

\section{CONCLUSIONES}

En una primera fase del análisis y con base en los resultados obtenidos se pudo evidenciar que los profesores del nivel primaria poseen en su mayoría tecnología móvil, ya que un $89 \%$ tienen una computadora portátil y un $78 \%$ una tableta; al mismo tiempo en dichos dispositivos el $91 \%$ se conecta a internet desde su casa, seguido de un $82 \%$ que se conecta en sus instituciones educativas, esto demuestra que los profesores cuentan con las Tecnologías de Información y Comunicación (TIC) para utilizarlas en sus diversas actividades académicas. Lo antes mencionado concuerda con lo expresado por Santoveña (2011), el cual hace énfasis en que la incorporación de las TIC es importante en la educación, ya que son un medio para apoyar al proceso de enseñanza y motivar el aprendizaje; así mismo internet es un servicio que permite tener acceso a un mundo de información el cual debe ser utilizado de una forma responsable, es por lo anterior que Galindo (2009), señala que el profesor independiente de su nivel educativo al estar utilizando tecnología adquiere un rol de ciudadano digital en el cual implementa las TIC de una manera ética dentro y fuera del salón de cla- 
ses.

En una segunda fase, se determinó el nivel de ciudadanía digital que tenían los profesores del nivel primaria en base al análisis de los diversos aspectos que integraban. Como resultado se pudo observar que el 70\% de los aspectos fueron valorados en un nivel medio y hubo un $20 \%$ de los aspectos que se valoró en un nivel bajo. Cabe destacar que los aspectos que salieron bajos hacen referencia a la falta de asesoramiento a los estudiantes para que puedan realizar búsquedas en internet confiables y seguras; así mismo, puedan citar dicha información de manera correcta en sus diversos trabajos académicos. Ante lo mencionado, autores como García, Gargallo, García y Sánchez (2012) argumentan que es importante ayudar a los estudiantes para convertirse en buscadores estratégicos, a través de la adquisición de habilidades relacionadas con la búsqueda, localización, recogida y selección de información a fin de que se conviertan en personas capaces de seguir un proceso sistemático y regulado para realizar búsquedas de información confiables y que les pueda servir para sus diversas tareas escolares.

Ante la importancia del profesor del nivel primaria como un elemento importante en el uso responsable de las TIC y con el fin de proponer cursos de capacitación para el desarrollo de la ciudadanía digital, se realizaron análisis estadísticos para determinar si hubo o no diferencia entre sus percepciones respecto a los diversos aspectos que integraban a la ciudadanía digital, con base en los resultados se pudo evidenciar que si hubo diferencia entre las percepciones de los profesores con base en la variable sexo; sin embargo, no hubo diferencia entre sus percepciones teniendo en cuenta las variables edad y grado máximo de estudios. Lo anterior nos puede indicar que para el diseño de un curso de capacitación en ciudadanía digital se recomienda que sea homogéneo, ya que la edad y grado académico fueron variables que no demostraron tener diferencia significativa; así mismo, el contenido se podría abordar desde un nivel básico, con el fin de consolidar las habilidades de búsqueda, diseminación y uso ético de la información.

Finalmente y consolidando lo antes mencionado, el Plan Estatal de desarrollo del estado de Yucatán (2018) en su objetivo 6.2.1.3.3, establece "promover los avances y beneficios de la ciencia y la tecnología en los municipios y en todos los niveles educativos", es por esto también importante capacitar a los profesores del nivel primaria para el desarrollo de su competencia y ciudadanía digital, ya que las pedagogías emergentes demandan el uso de tecnologías en entornos presenciales y no presenciales de una forma responsable. No hay que olvidar que en la actualidad la educación utiliza plataformas tecnológicas e internet.

Por lo antes mencionado, los profesores no pueden es- tar ajenos a los avances tecnológicos y mucho menos a la formación continua para el uso de las TIC de una manera responsable, ya que ellos son la mediática principal para la formación integral de los estudiantes en cualquier nivel educativo. 


\section{지 P U B L I C A N D O I S S N $133900-93004$}

\section{REFERENCIAS BIBLIOGRÁFICAS}

Alejandro, N., Benítez, M. y Ortiz, G. (2015). Reseña ciudadanía digital. Entre la novedad del fenómeno y las limitaciones del concepto. Revista Economía, Sociedad y Territorio, 15 (49). Recuperado de: h t t p : / / w w w . scielo.org. $\mathrm{mx} / \mathrm{scielo}$.php? script $=$ sci_arttex t\&pid=S1405-84212015000300010

Alva, A. (2019). Escenarios y desafíos de la ciudadanía digital en México Revista Mexicana de Ciencias Políticas y Sociales. LXV (238), 81-105. Recuperado de: http://www.revistas.unam. $\mathrm{mx} /$ index.php/rmcpys/article/view/68337/63872

Antúnez, G., González, K., Soler, Y., Rodríguez, S. y J. Hauß (2013). Resultados y experiencias en la enseñanza de las matemáticas en modalidad: Blended learning. Recuperado de: https:// bit.ly/3nNffeH

Arnau, J. (1995). Metodologías cuantitativas en la investigación psicológica. Barcelona,

Espa ñ a: Experimental.

Ávila, P. (2016). Construcción de ciudadanía digital: un reto para la Educación. Suplemento SIGNOS, EDA. 2015 XI. Conferencia Internacional Guide. Recuperado de: https://p3.usal. edu.ar/index.php/supsignosead/article/down$\operatorname{load} / 3666 / 4533$

Cárdenas, M. (2018). ¿Qué sabemos sobre ciudadanía digital en México? Reporte y procesamiento, del anexo técnico para diagnosticar el nivel de conocimiento que, tienen tanto alumnos y alumnas como docentes de escuelas públicas, en Ciudadanía Digital. México: SEP y @prende 2.0. Recuperado de: https://www.gob.mx/cms/uploads/attachment/ file/418090/Informe_Ciudadania_Digital_-_ WEB-compressed.pdf

Casal, J. y Mateu, E. (2003). Tipos de muestreo. Revista de Epidemiología y Medicina Preventiva, 1(1), 3-7.

Casas, J., Repullo, J. y Donaldo, J. (2003). La encuesta como técnica de investigación. Elaboración de cuestionarios y tratamiento estadístico de los datos. Atención Primaria, 31(8), 527-538. Recuperado de: https://www.sciencedirect.com/science/article/pii/S0212656703707288
Cea D’Ancona, M. (2001). Metodología cuantitativa. Estrategias y técnicas de investigación social. Madrid, España: Síntesis.

Cobo, C. (2019). Ciudadanía digital y educación: nuevas ciudadanías para nuevos entornos. Revista Mexicana de Bachillerato a Distancia, 11 (21), 1-8. Recuperado de: http://revistas.unam. $\mathrm{mx} /$ index.php/rmbd/article/view/68214

Cota, G. (2017). La competencia digital en los futuros docentes en educación primaria. Recuperado de: http://www.conisen.mx/memorias/memorias/1/ C200117-R151.docx.pdf

Creswell, J. (2012). Educational research: planning, conducting, and evaluating

quantitative and qualitative research. Boston: Pearson.

Galindo, J. (2009). Ciudadanía digital. Revista Signo y Pensamiento. XXVIII (54), 164- 173. Recuperado de: https://www.researchgate.net/publication/43236090_Ciudadania_digital

García, J., Gargallo, B., García, A. y Sánchez, F. (2012). Nuevos modos de aprendizaje en el contexto de la sociedad del conocimiento. XXXI Seminario Interuniversitario de Teoría de la Educación: Sociedad del Conocimiento y Educación. Plasencia: UNED.

García-Valcárcel, A., Basilotta, V. y Mulas, I. (2016). Fomentando la ciudadanía digital mediante un proyecto de aprendizaje colaborativo entre escuelas rurales y urbanas para aprender inglés Profesorado. Revista de Currículum y Formación de Profesorado, 20 (3), 549-581 Recuperado de: http s : / / recyt.fecyt.es/index.php/profesorado/article/ view/54613

George, D. y Mallery, P. (2003). SPSS for Windows step by step: A simple guide and reference, 11.0 update (4thed.). Boston: Allyn \& Bacon.

Hernández, R. (2017). Impacto de las TIC en la educación: Retos y Perspectivas. Revista Propósitos y Representaciones. 5 (1), 325 -347. Recuperado de: https://dialnet.unirioja.es/descarga/articulo/5904762.pdf 
Hernández, R., Fernández, C. y Baptista, M. (2013). Metodología de la Investigación (6.a ed.). Ciudad de México, México: McGraw-Hill.

Instituto Nacional Electoral [INE] (2020). Impulsa INE Yucatán participación ciudadana a través de redes sociales. Instituto Nacional Electoral. Recuperado de: https://centralelectoral. ine. $\mathrm{mx} / 2020 / 05 / 08 /$ impulsa-ine-yucatan-participacion-ciudadana-traves-redes-sociales/

International Society For Technology in Educacion [ISTE] (2017). Standars for educators. Recuperado de: http://www.iste.org/standards/for-educators

Isaac, S. y Michael, W. (1995). Handbook in Research and Evaluation. San Diego, United States: EDITS Publishers.

Lozano, A. y Fernández, J. (2019). Hacia una educación para la ciudadanía digital crítica y activa en la universidad, (18) 1, 175-187. Recuperado de: https://relatec.unex.es/article/view/3390/2350

Peña, P. (s/f). Orientaciones de ciudadanía digital para la formación ciudadana. Ministerio de Educación, República de Chile. Recuperado de: https://www.cpeip.cl/wp-content/uploads/2018/04/orientaciones-de-ciudadania-digital-para-la-formacion-ciudadana-web.pdf

Plan Estatal de Desarrollo de Yucatán [PEDY] (2018). Gobierno del Estado de Yucatán. Mérida, Yucatán, México. Recuperado de: http://www.yucatan.gob.mx/docs/transparencia/ped/2012_2018/ PED_2012_2018.pdf

Prieto, E. (2008). El papel del profesorado en la actualidad. Su función docente y social. Foro de Educación, 6 (10), 325-345. Recuperado de: https://dialnet.unirioja.es/servlet/articulo?codigo $=2907073$

Organización de la Naciones Unidas para la Educación, la Ciencia y la Cultura [UNESCO] (2014). Fostering Digital Citizenship through Safe and Responsible Use of ICT: A Review of Current Status in Asia and the Pacific as of December 2014. Bangkok. Recuperado de: https:// en.unesco.org/sites/default/files/sru-ict_mapping_ report_2014.pdf

Organización de la Naciones Unidas para la Educación, la Ciencia y la Cultura [UNESCO] (2016). Competencias y estándares TIC desde la dimensión pedagógica: Una perspectiva desde los niveles de apropiación de las TIC en la práctica educativa docente. Recuperado de: http://www.unesco. org/new/fileadmin/MULTIMEDIA/FIELD/Santiago/pdf/Competencias-estandares-TIC.pdf

Santoveña, S. (2011). Procesos de comunicación a través de entornos virtuales y su incidencia en la formación permanente en red. Revista de Universidad y Sociedad del Conocimiento, 8 (1), 93110. Recuperado de https://core.ac.uk/download/ pdf/39015473.pdf

Stramiello, C. (2005). ¿Una educación humanista hoy? Revista Iberoamericana de Educación. (36/8), 1-5. Recuperado de: https://rieoei.org/RIE/article/ view/2777

Vincezi, A. \& Tudesco, F. (2009). La educación como proceso de mejoramiento de la calidad de vida de los individuos y de la comunidad. Revista Iberoamericana de Educación. (49/7). Recuperado de: https://rieoei.org/RIE/article/view/2047

Walter, G. (2015). Reseña. Ciudadanía digital. Entre la novedad del fenómeno y las $\quad 1$ i m i t a ciones del concepto. Revista Economía, Sociedad y Territorio, 15 (49). Recuperado de: h t t p : / / www.scielo.org.mx/scielo.php?script $=$ sci_arttext\&pid=S1405-84212015000300010 